\title{
A selective impairment in the phonological output lexicon
}

\author{
T. Imamura, A. Yamadori ${ }^{1}$, M. Aoki, R. Mochizuki and K. Kogure \\ Department of Neurology, Institute of Brain Diseases, Tohoku University School \\ of Medicine, Sendai, ${ }^{1}$ Hyogo Institute for Aging Brain and Cognitive Disorders \\ Himeji, Japan \\ Correspondence to: T. Imamura, Division of Behavioral Neurology, Department \\ of Clinical Neurosciences, Hyogo Institute for Aging Brain and Cognitive Disorders, \\ 520, Saisyo-Ko, Himeji, Hyogo 980, Japan
}

\begin{abstract}
It has been proposed that words are stored in a functional structure which consists of a semantic system and modality-specific input and output lexicons. We report a patient with disturbed word finding for given letters, but not for specific categories. The patient had difficulty in phonological similarity judgements of pictures, but performed normally on semantic similarity judgements. We conclude that this patient's impairments stemmed from a disturbance restricted to the subsystem for active judgement and selection of representations in the phonological output lexicon.
\end{abstract}

Keywords: Anomic aphasia - Lexical knowledge - Semantic system - Word finding difficulty - Word store

\section{INTRODUCTION}

A recent theoretical framework of cognitive neuropsychology assumes that knowledge about words is stored in a functional architecture which consists of one central semantic system and modality-specific input and output lexicons (Caramazza and Hillis, 1990; Hillis and Caramazza, 1991). According to this theory, a restricted deficit in each component, or in one subsystem of each component, may manifest a particular language disturbance. Several clinical observations of aphasics support this assumption. For example, disturbance of abstract or categorical attitude (Goldstein, 1948; Yamadori and Albert, 1973; Fujino et al., 1991), and category-specific naming and comprehension impairments (see Gainotti, 1990, for review) can be attributed to the disturbances in certain subsystems of the semantic component. Caramazza and Hillis (1990), on the other hand, described patients whose deficit is restricted to phonological output lexicon.

We report here a patient with word finding difficulty. Our findings suggest that this patient's phonological output lexicon was disturbed in a different way from previous reports.

\section{CASE REPORT}

A 35-year-old right-handed female university graduate was admitted to the gynaecology department because of severe dehydration and hemoconcentration caused by extrinsic gonadotropin (ovarian hyperstimulation syndrome). One day after admission, she suddenly developed difficulty in speech and writing. She was rehydrated but her language disorder persisted, and she presented to us 1 month later.

She was alert, orientated in time and space and cooperative. General neurological examination was normal. Magnetic resonance imaging (MRI) exhibited patchy infarction in left lateral temporal and insular cortices (Fig. 1). A cerebral angiogram revealed no vascular occlusion or stenosis.

On neuropsychological examination, there was no agnosia or apraxia. Her scores on the Wechsler Adult Intelligence Scale (WAIS) were excellent (Table I). She repeatedly complained of a difficulty in word retrieval, but conversational speech was fluent with no articulatory disturbance or paraphasia. She repeated and executed multiple step verbal orders with no difficulty. Pointing of 50 pictures of living and non-living items were all accurate and carried out with no hesitation. Pointing span was five in seven objects. There was no abnormality in writing or reading of letters, words and sentences. On confrontation naming of 90 pictures and objects (30 small man-made objects, 12 large man-made objects, 11 pictures indicating some action, 14 body parts, eight living things and 15 


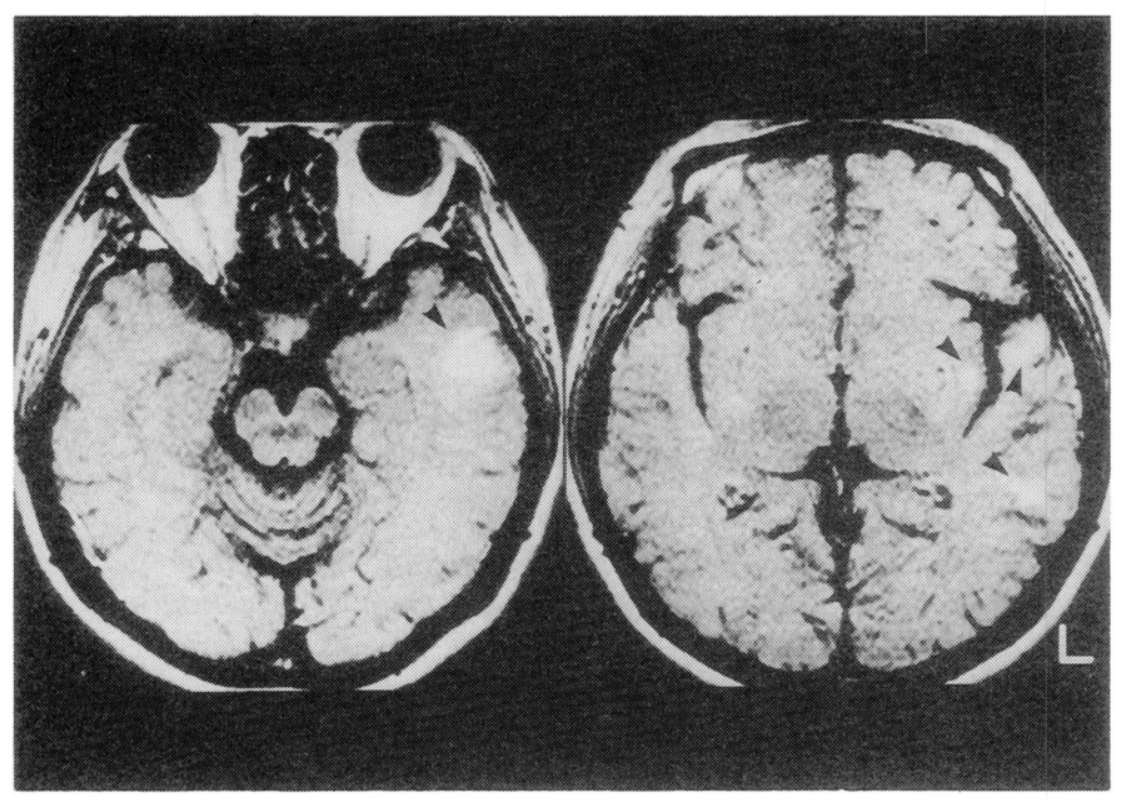

FIG. 1. Multiple spotty infarcations (arrowheads) were enhanced by contrast material on magnetic resonance T1-weighted images (TR 607, TE 30).

foods including vegetables and fruits), her responses were accurate and fluent except for three hesitations and one verbal paraphasia. She performed word finding tasks adequately when given a category (bird, 14/min; animal, $12 / \mathrm{min}$; flower, $15 / \mathrm{min}$ ), but when given an initial Kana letter, she retrieved only 5, 2, 6 and 4 words per minute, respectively on four trials. She always pronounced a few adequate words at the beginning of trials, but showed great difficulty in further production. Normal subjects tend to retrieve less words with initial letter than with category (Rosen, 1980; Fukuzawa et al., 1988). We, however, judged her word finding with given initial Kana was insufficient when compared with the good performance on other tasks, and her educational level.

Her objective word finding difficulty became evident in a phonologically conditioned task. We performed further

TABLE I. Scores on Wechsler Adult Intelligence Scale

\begin{tabular}{lrlc}
\hline & $\begin{array}{c}\text { Scaled } \\
\text { score }\end{array}$ & $\begin{array}{c}\text { Scaled } \\
\text { score }\end{array}$ \\
\hline Information & 12 & Digit symbol & 17 \\
Comprehension & 11 & Picture completion & 13 \\
Similarities & 15 & Block design & 13 \\
Arithmetic & 12 & Picture arrangement & 10 \\
Digit span & 9 & Object assembly & 11 \\
Vocabulary & 14 & & \\
Verbal IQ & 113 & Performance IQ & 120 \\
\hline
\end{tabular}

studies to evaluate her phonological and semantic processing capacity of words.

\section{METHODS}

Nine normal volunteers matched for age and educational history served as controls.

We conducted two experimental investigations, using semantic and phonological similarity judgement tasks. In semantic similarity judgement, visual stimuli consisted of 18 arrays of six line drawn pictures. In each array, one target picture belonged to the same category as an auditory stimulus word. Subjects were instructed to point to a picture in the same category as the stimulus word. In phonological similarity judgement, 12 arrays of six line drawn pictures were presented as visual stimuli (Fig. 2). The target picture of each array had the same name as an auditory stimulus word except one syllable. The other five pictures were distractors, and all their names had no common phonemes to the stimulus word. Three of them had the same, but the other two had different, numbers of syllables to the stimulus word (see Fig. 2 for example). Subjects were instructed to point to the picture the name of which sounded closest to the auditory stimulus word.

In each task, an examiner pronounced a stimulus word, and then presented an array of pictures. Response time from visual presentation to pointing was measured manually. One practice task was performed in both experiments. We had confirmed that both the patient and the normal controls could name all the pictures correctly without hesitation, before the actual performance. 

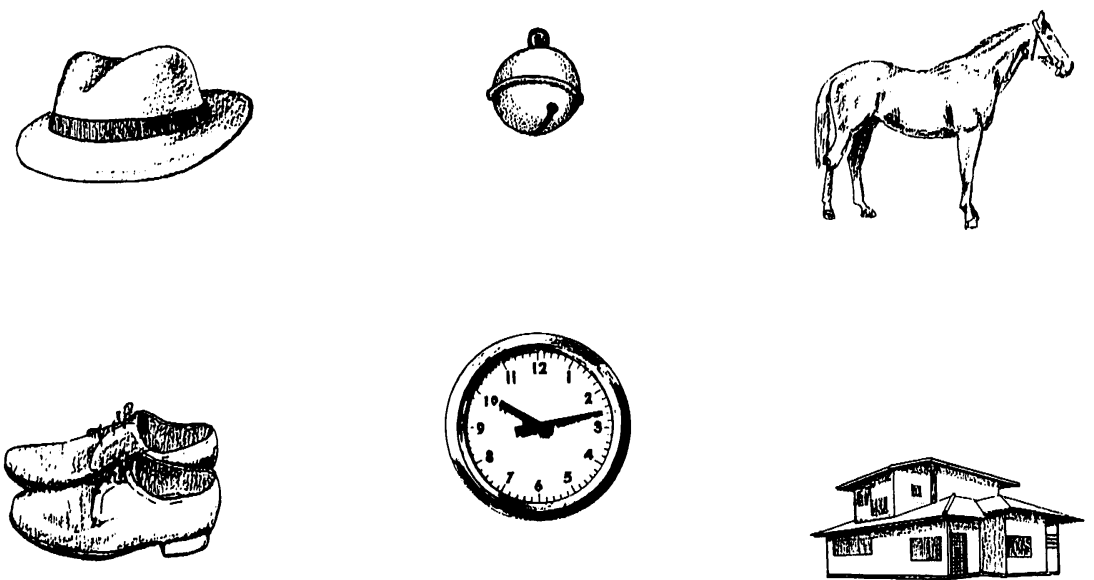

FIG. 2. An example of a visual stimulus in phonological similarity judgement. On this task, the stimulus word is [yama] (which means mountain in Japanese). The target picture is horse which is [uma] in Japanese. The other five pictures, bell, shoes, house, hat and clock which are [suzu], [kutsu], [ie], [bousi], and [tokei], respectively, are distractors.

\section{RESULTS}

All normal controls performed both tasks correctly with no hesitation. The mean ( \pm S.D.) response time of normals was $2.2( \pm 0.5) \mathrm{s}$ in category judgement, and 3.8 $( \pm 1.8) \mathrm{s}$ in phonological judgement, and their response time was not above 5 and $10 \mathrm{~s}$, respectively. Thus, we determined the normal range as up to $5 \mathrm{~s}$ in categorical judgement, and up to $10 \mathrm{~s}$ in phonological judgement. The patient's performances in categorical judgement were also correct with a prompt manner, and her response times were all within normal range. In phonological judgement, however, she exhibited occasional struggles and hesitations. The response time was longer than $10 \mathrm{~s}$ in three of 12 trials, though her final pointing was correct.

\section{DISCUSSION}

The patient complained of difficulty in word finding, but objective abnormality was so slight that it may be easily overlooked by formal assessment for aphasia. The disturbance, however, became evident when she was required to retrieve words with a given initial letter. Perret (1974) attributed this pattern of word finding disturbance to the deficits in the ability to suppress habitual behavior and to adapt to unusual situations caused by frontal lobe dysfunction. However, this interpretation seems to be inappropriate in this patient because the high scores on most subtests of WAIS (Table I) would argue against frontal lobe dysfunction. The excellent performance on verbal subtests excludes the possibility that semantic processing impairments disrupted her word retrieval. It is also unlikely that her word finding difficulty was secondary to expressive language impairments, because no signs of articulatory disturbance or literal paraphasia were present.

We have investigated the patient's impairment in relation to theories of cognitive neuropsychology (Caramazza and Hillis, 1990; Hillis and Caramazza, 1991). Let us consider the processes involved in performing categorical word finding (Fig. 3A). First, an examiner pronounces a word indicating a category. This stimulus is linked, via auditory analysis, to the equivalent representation in the phonological input lexicon. This representation activates the corresponding semantic representation. The given category is therefore accepted into the semantic system, and representations of adequate categorical word lists prepared. These semantic representations activate equivalent output representations in the phonological output lexicon, and words are pronounced one by one in the final process. The scheme is different when given an initial Kana letter (Fig. 3B). An examiner gives a syllable as auditory stimulus of this task because every initial Kana corresponds to one syllable in Japanese writing. The processes of auditory analysis convert this stimulus to the equivalent neural information, but it would not be linked to the phonological input lexicon or semantic system, because there is no corresponding representation for each syllable. Therefore, the phonological output lexicon directly accepts this neural information and prepares output representations of adequate word list. Finally, words with a given initial syllable (or Kana letter) are pronounced one by one.

The patient's word finding was inadequate only when given initial Kana. Thus, her deficit exists in the processes shown in Fig. 3B. The information of a given syllable, however, seemed to be accepted immediately by the phonological output lexicon, because she could produce 


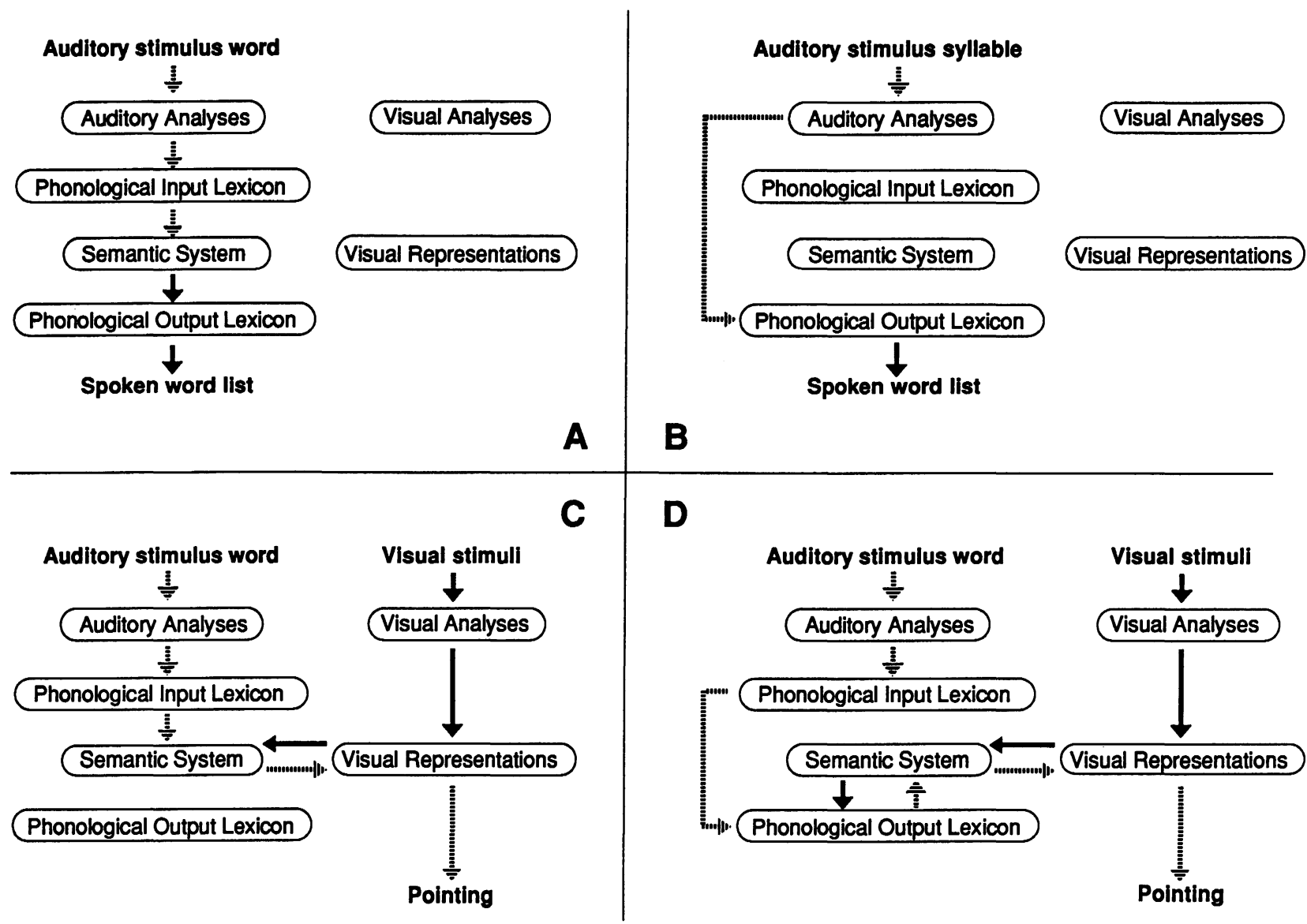

FIG. 3. Schematic representations of the processes in (A) categorical and (B) phonological word finding, and (C) categorical and (D) phonological similarity judgement task. Striped and black arrows represent the flow of single and multiple informations, respectively. Orthographic lexicons are omitted in this figure.

several adequate words soon after an examiner pronounced the syllable. The expressive processes from phonological output representations to word pronunciation were also intact as mentioned above. Therefore, her deficit seemed to be within the phonological output lexicon. On categorical word finding, representations of word list are prepared in a semantic system, and the phonological output lexicon works as a "relay station". On the other hand, when given initial Kana, phonological output lexicon itself selects and prepares adequate representations of word list according to the given syllable. A disturbance in the process of active judgement and selection within phonological output lexicon seems to explain best the patient's impairment.

In the categorical similarity judgement task (Fig. 3C), an auditory stimulus word activates the corresponding phonological, and then semantic, representation. Presented pictures are linked, via visual analyses, to the equivalent visual representations, and they activate corresponding semantic representations. Then, all the activated representations in the semantic system are compared to select the equivalent one to the target picture. The selected semantic representation reactivates the corresponding visual representation, and finally, the target picture is pointed to. The phonological output lexicon makes no contribution to this task, and the patient performed it satisfactorily.

In the phonological similarity judgement task (Fig. 3D), the auditory stimulus word is linked to the equivalent phonological input representation, and then it would directly activate the corresponding representation in the phonological output lexicon, because this task needs no semantic processing of the stimulus word. Presented pictures are linked to the equivalent visual representations. They activated the corresponding semantic, and then the output, representations. All the activated representations are compared phonologically, and the equivalent one to the target picture is selected in the phonological output lexicon. This representation reactivates the corresponding semantic, and then visual, representation, which makes adequate pointing possible. In the processes of performing this task, the interactions of visual and semantic representations are identical to the processes in categorical simi- 


\section{A SELECTIVE IMPAIRMENT IN THE PHONOLOGICAL OUTPUT LEXICON}

larity judgement. Activation from the semantic system to the phonological output lexicon also occurs in categorical word finding. Direct linkage from phonological input to output lexicon is common to the process in simple repetition. All these processes were intact in this patient. Her performance of phonological similarity judgement is, however, insufficient, and the active judgement and selection of representation in phonological output lexicon is again essential to this task.

In conclusion a deficit of the subsystem for active judgement and selection within the phonological output lexicon resulted in the patient's poor performance. This selective impairment also explains the dissociation between her subjective complaint and the adequate performance on naming and repetition. In the tasks in formal language assessments, such as confrontation naming and sentence repetition, phonological output lexicon mostly works as a "relay station". In daily conversation, however, active judgement and selection of phonological output representations are needed because the phonological characteristics of words are essential in preparing the rhythm and euphony of speech.

Magnetic resonance imaging showed lesions in left lateral temporal and insular cortices. This distribution was different from the patients of Caramazza and Hillis (1990), where the lesions were mainly in the left parietal lobe. Cerebral dysfunction of this patient, however, may not be lim- ited to the MRI documented lesions as the pathology was hemorrhagic.

\section{REFERENCES}

Caramazza A and Hillis AE (1990) Where do semantic errors come from? Cortex, 26, 95-122.

Fujino H, Iwakura T and Shibuya N (1991) A case of amnesic aphasia due to impairment of so-called "categorical attitude". Higher Brain Function Research, 11, 230-236 (in Japanese).

Fukuzawa K, Tatsumi I and Sasanuma S (1988) Impairment of word fluency in the patients with dementia. Higher Brain Function Research, 8, 243-250 (in Japanese).

Gainotti G (1990) The categorical organization of semantic and lexical knowledge in the brain. Behavioural Neurology, 3, 109-115.

Goldstein K (1948) Language and Language Disturbances, pp. 246-291. Grune \& Stratton, New York.

Hillis AE and Caramazza A (1991) Category-specific naming and comprehension impairment: a double dissociation. Brain, 114, 2081-2094.

Perret E (1974) The left frontal lobe of man and the suppression of habitual responses in verbal categorical behaviour. Neuropsychologia, 12, 323-330.

Rosen WG (1980) Verbal fluency in aging and dementia. Journal of Clinical Neuropsychology, 2, 135-146.

Yamadori A and Albert ML (1973) Word category aphasia. Cortex, 9, 112-125.

(Received 20 February 1993; accepted 30 March 1993) 


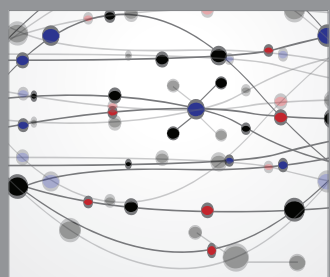

The Scientific World Journal
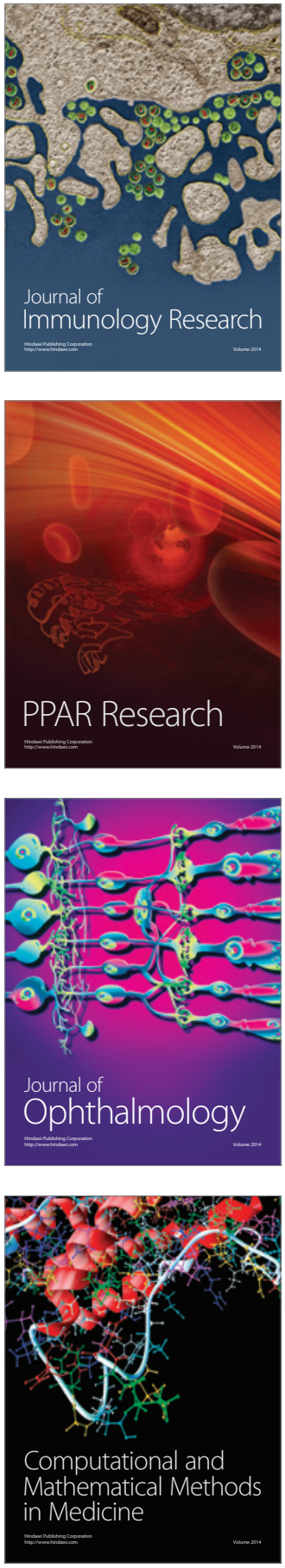

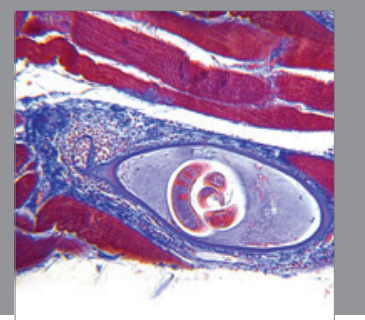

Gastroenterology

Research and Practice
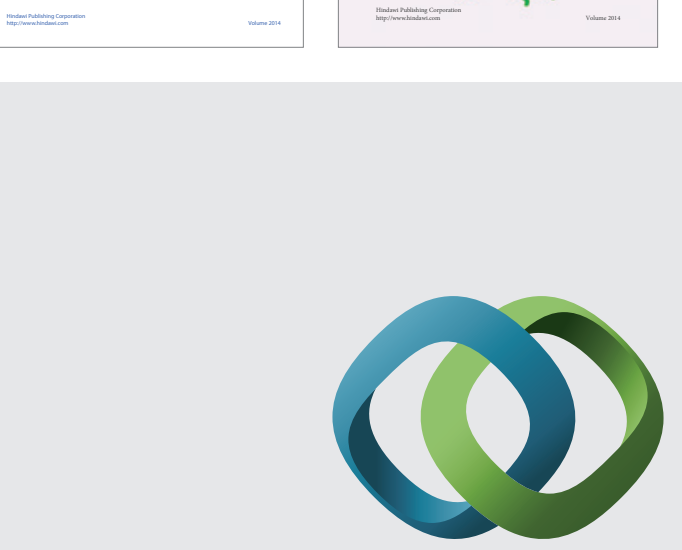

\section{Hindawi}

Submit your manuscripts at

http://www.hindawi.com
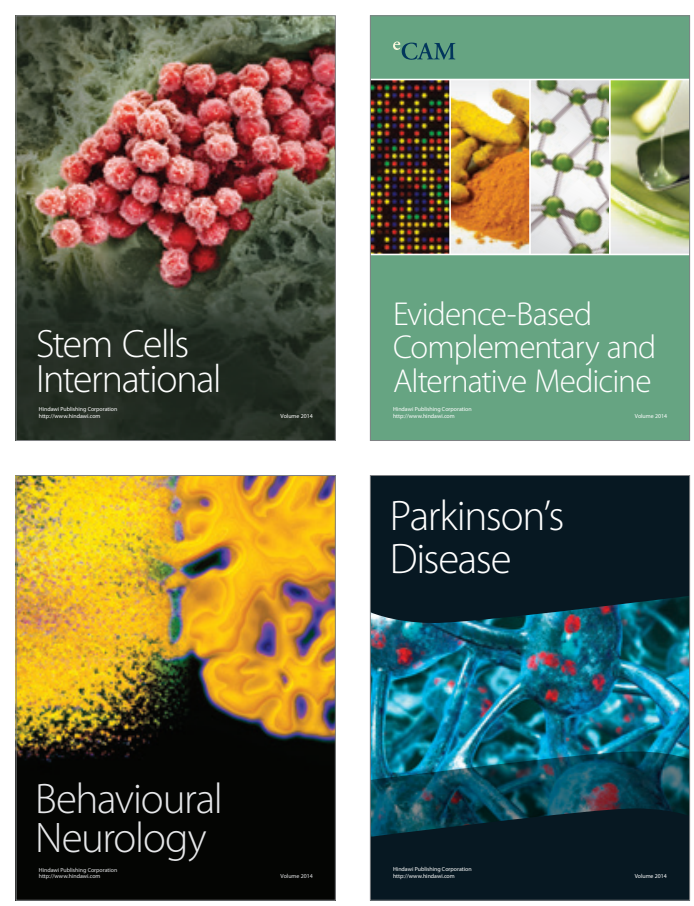

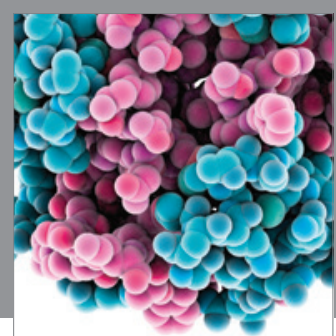

Journal of
Diabetes Research

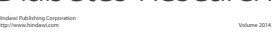

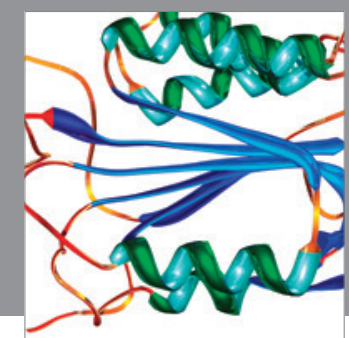

Disease Markers
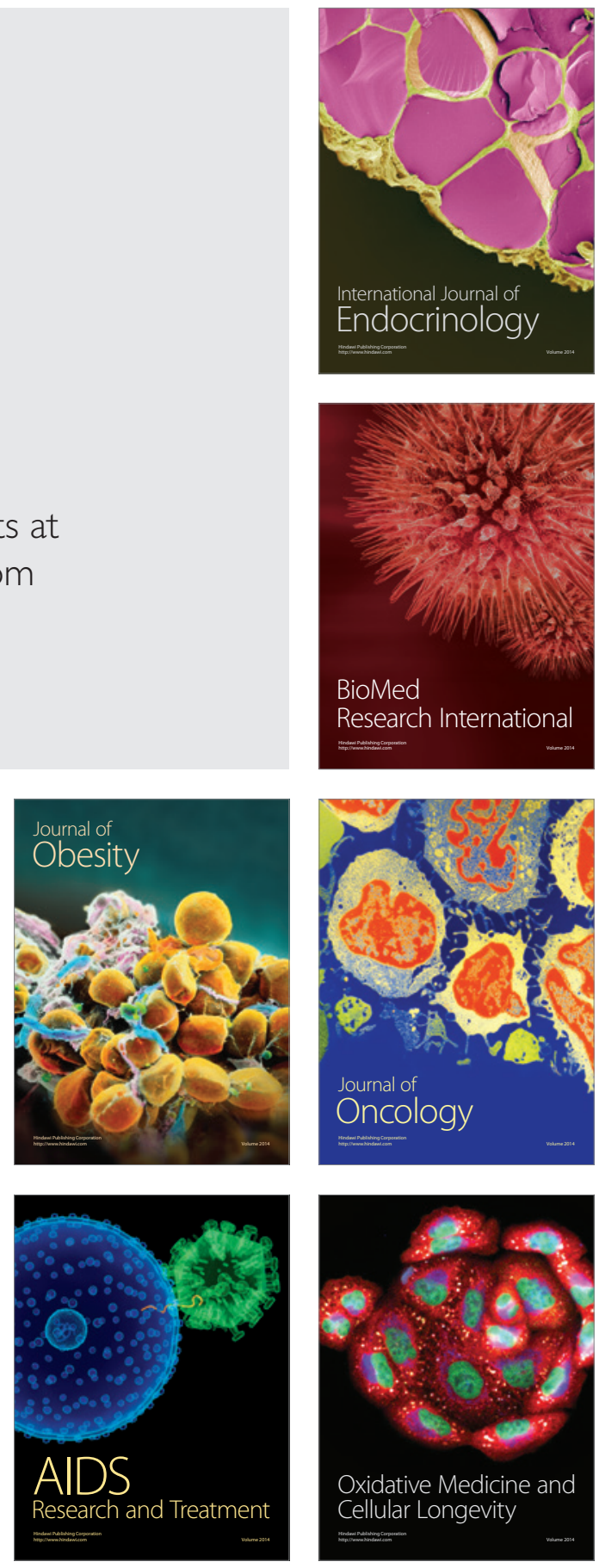